\title{
Exact supersymmetry in the nonrelativistic hydrogen atom
}

\author{
R. D. Tangerman \\ National Institute for Nuclear Physics and High Energy Physics (NIKHEF-K), \\ P.O. Box 41882, 1009 DB Amsterdam, The Netherlands \\ and \\ J. A. Tjon \\ Institute for Theoretical Physics, \\ Princetonplein 5, P.O. Box 80.006, 3508 TA Utrecht, The Netherlands
}

\begin{abstract}
We consider a Pauli particle in a Coulomb field. The supersymmetric Hamiltonian is constructed, by explicitly giving the two supercharges $Q_{1}$ and $Q_{2}$ in the full three-dimensional space and which together with the Hamiltonian, are shown to constitute an $S(2) \mathrm{su}^{-}$ peralgebra. This offers an alternative way of grouping the energy eigenstates into irreducible representations of this symmetry group of the Hamiltonian.
\end{abstract}

PACS numbers: 03.65.-w,11.30.Pb 


\section{Introduction}

In recent years considerable attention has been paid to supersymmetric (SUSY) quantum mechanics (for a review see [1] and the references therein). An interesting suggestion in this context has been made by Kostelecký and Nieto [2], that SUSY may be realized in atomic systems as a good symmetry to characterize the spectrum of alkali-metal atoms. In the discussion of SUSY quantum mechanics one usually considers one-dimensional systems. For the simplest case $(\mathrm{N}=2)$ the SUSY Hamiltonian is expressed in terms of two operators $Q_{1}$ and $Q_{2}$, called supercharges, obeying the $\mathrm{S}(2)$ product rules

$$
\begin{aligned}
& {\left[Q_{1}, \tilde{H}\right]=\left[Q_{2}, \tilde{H}\right]=0,} \\
& \left\{Q_{1}, Q_{2}\right\}=0 \\
& \left\{Q_{1}, Q_{1}\right\}=\left\{Q_{2}, Q_{2}\right\}=\tilde{H},
\end{aligned}
$$

with $\tilde{H}=f(H)$ being an invertable function of the original Hamiltonian $H$.

Applying it to the case of the hydrogen atom [2, 3], the corresponding onedimensional superpotentials [4] can be determined by considering the radial part of the Schrödinger equation. In higher dimensions superalgebras can explain accidental spectrum degeneracies as has been shown for the threedimensional harmonic oscillator [5]. D'Hoker and Vinet have discovered a supersymmetry for a spin- $\frac{1}{2}$ particle in the presence of a dyon field [6]. However, for this they need the introduction of an additional particle. For the hydrogen problem it is expected that an explicit form of a SUSY Hamilto-

nian in three dimensions does exist. We indeed show in this paper how the 
supercharges can be obtained in this case, by just using the spin degrees of freedom of the electron. We stress the fact that no extra particles are needed in our formalism.

In the next section we construct a $N=2$ supersymmetry for the Coulomb potential. Use is made of the well-known $S O(4)$ symmetry. The multiplets are identified in section 3 and the connection is made with radial supersymmetries. Finally, in section 4 we discuss the possible implications for other systems, in particular the relativistic spin- $\frac{1}{2}$ particle in a Coulomb field.

\section{Construction of the charges}

The accidental degeneracies of the hydrogen atom bound state spectrum can be ascribed to an $S O(4)$ symmetry [7] of its Hamiltonian

$$
H=\frac{1}{2} \mathbf{p}^{2}-\frac{1}{r} .
$$

Apart from the obvious $S O(3)$-invariance, generated by the angular momenta

$$
\mathbf{L}=\frac{1}{2}(\mathbf{x} \times \mathbf{p}-\mathbf{p} \times \mathbf{x}),
$$

it has three additional constants of the motion, which together form the Runge-Lenz vector

$$
\mathbf{A}_{0}=\frac{1}{2}(\mathbf{p} \times \mathbf{L}-\mathbf{L} \times \mathbf{p})-\frac{\mathbf{x}}{r} .
$$

Only after proper normalization

$$
\mathbf{A} \equiv \begin{cases}\frac{\mathbf{A}_{0}}{\sqrt{-2 H}} & \text { if } E<0 \\ \mathbf{A}_{0} & \text { if } E=0 \\ \frac{\mathbf{A}_{0}}{\sqrt{2 H}} & \text { if } E>0\end{cases}
$$


one obtains a closed algebra. For the bound states $(E<0)$ the product rules are

$$
\begin{aligned}
& {\left[L_{i}, L_{j}\right]=i \varepsilon_{i j k} L_{k},} \\
& {\left[L_{i}, A_{j}\right]=i \varepsilon_{i j k} A_{k},} \\
& {\left[A_{i}, A_{j}\right]=i \varepsilon_{i j k} L_{k} .}
\end{aligned}
$$

They form the defining relations of an $S O(4)$ algebra円. The two Casimir operators

$$
\begin{gathered}
C_{1}=\mathbf{L}^{2}+\mathbf{A}^{2}, \\
C_{2}=\mathbf{A} \cdot \mathbf{L},
\end{gathered}
$$

in the present realization assume the form

$$
\begin{gathered}
C_{1}=-\frac{1}{2 H}-1, \\
C_{2}=0 .
\end{gathered}
$$

The general versions of these relations, being valid at all energies, are

$$
\begin{gathered}
\mathbf{A}_{0}^{2}=2 H\left(\mathbf{L}^{2}+1\right)+1, \\
\mathbf{A}_{0} \cdot \mathbf{L}=\mathbf{L} \cdot \mathbf{A}_{0}=0 .
\end{gathered}
$$

To construct the supercharges for the Coulomb problem we also include the spin degrees of freedom. As a consequence the symmetry algebra is enlarged with the $S U(2)$ generators $\mathbf{S}=\frac{1}{2} \sigma$, resulting into a $S O(4) \times S U(2)$ symmetry group for the bound states. Usually one chooses to diagonalize the set of observables $H, \mathbf{J}^{2}, \mathbf{L}^{2}$ and $J_{3}$. Following Biedenharn and Louck [8], the two operators $\mathbf{J}^{2}$ and $\mathbf{L}^{2}$ can be replaced by a single scalar $\mathcal{K}$, defined by

$$
\mathcal{K} \equiv-(2 \mathbf{S} \cdot \mathbf{L}+1)
$$

\footnotetext{
${ }^{1}$ In contrast, the scattering states $(E>0)$ are $S O(3,1)$-multiplets.
} 
We have

$$
\left\{\begin{array} { l } 
{ \mathbf { L } ^ { 2 } = \mathcal { K } ( \mathcal { K } + 1 ) } \\
{ \mathbf { J } ^ { 2 } = \mathcal { K } ^ { 2 } - 1 }
\end{array} \Rightarrow \left\{\begin{array}{l}
\ell(\kappa)=|\kappa|+\frac{1}{2}(\operatorname{sgn} \kappa-1) \\
j(\kappa)=|\kappa|-\frac{1}{2}
\end{array}\right.\right.
$$

where $\kappa$ takes the values $\{ \pm 1, \pm 2, \ldots\}$. It is clear that states with fixed $j$ and $\ell=j \pm \frac{1}{2}$ correspond to equal $|\kappa|$ but with $\operatorname{sgn} \kappa= \pm 1$.

A $Z_{2}$-grading can now be introduced in the Hilbert space of states by classifying the states to be even or odd with respect to the parity operator

$$
P_{\kappa} \equiv \frac{\mathcal{K}}{|\kappa|}
$$

i.e. having eigenvalues $\pm \operatorname{sgn} \kappa$ respectively. Equally, linear operators can be assigned a grade. An operator is even, if it commutes with $P_{\kappa}$, whereas operators anti-commuting with $P_{\kappa}$ are called odd. As an example of an even operator we mention $H$, following from the fact that $\mathcal{K}$ is built from symmetry operators of $H$. Of course, $\mathcal{K}$ itself is even as well. To find odd operators, we make use of the following theorem.

Theorem. Suppose $\mathbf{V}$ is a vector with respect to the orbital angular momentum $\mathbf{L}$ that is also perpendicular to $\mathbf{L}$

$$
\begin{aligned}
& {\left[L_{i}, V_{j}\right]=i \varepsilon_{i j k} V_{k},} \\
& \mathbf{L} \cdot \mathbf{V}=\mathbf{V} \cdot \mathbf{L}=0,
\end{aligned}
$$

then $\mathcal{K}$ anticommutes with the $\mathbf{J}$-scalar $\mathbf{S} \cdot \mathbf{V}$.

For a proof see Ref. [8]. This theorem supplies us with odd operators $\mathbf{S} \cdot \mathbf{V}$, with $\mathbf{V}$ equal to for example $\mathbf{p}, \mathbf{r}$ or $\mathbf{A}_{0}$. 
Considering the square of the odd operator $\mathbf{S} \cdot \mathbf{A}_{0}$, it is readily shown that

$$
\left(\mathbf{S} \cdot \mathbf{A}_{0}\right)^{2}=\frac{1}{2} H \mathcal{K}^{2}+\frac{1}{4}
$$

where Eq. (10) has been used. Restricting ourself to the subspace $\mathcal{H}_{k}$ of fixed $|\kappa| \equiv k$, this can be rewritten as

$$
2\left(\frac{\mathbf{S} \cdot \mathbf{A}_{0}}{k}\right)^{2}=H+\frac{1}{2 k^{2}} .
$$

We now define the supercharge

$$
Q_{1} \equiv \frac{\mathbf{S} \cdot \mathbf{A}_{0}}{k}
$$

and the shifted Hamiltonian

$$
\tilde{H} \equiv H+\frac{1}{2 k^{2}}
$$

Eq. (17) can then be identified with the $S(2)$-product rule $\left\{Q_{1}, Q_{1}\right\}=\tilde{H}$. Moreover, using the fact that $Q_{1}$ is odd and $H$ is even, we readily identify the second charge as

$$
Q_{2} \equiv i Q_{1} P_{\kappa}=\frac{i}{k^{2}}\left(\mathbf{S} \cdot \mathbf{A}_{0}\right) \mathcal{K}
$$

thereby completing the $S(2)$ symmetry algebra.

Instead of working in the Hermitian representation of $S(2)$ as described above, one often uses the odd ladder operators

$$
Q_{ \pm} \equiv \frac{1}{\sqrt{2}}\left(Q_{1} \pm i Q_{2}\right)=\frac{1}{\sqrt{2} k}\left(\mathbf{S} \cdot \mathbf{A}_{0}\right)\left(1 \mp P_{\kappa}\right)
$$

Note that for the even states $Q_{+}=0$ and $Q_{-}=\frac{\sqrt{2} \mathbf{S} \cdot \mathbf{A}_{0}}{k}$, whereas for the odd states their roles are reversed. 


\section{The hydrogen spectrum revisited}

We now turn to discuss the implications of the constructed $S(2)$ symmetry on the spectrum. The consequence of a symmetry group of a Hamiltonian is that the energy eigenspaces consist of irreducible representations of it. The irreducible representations of $S(2)$ are known to be either one- or twodimensional. Consider the energy eigenstates of the Pauli particle, given by

$$
\phi_{E \ell(\kappa) j(\kappa) m}(r, \theta, \varphi)=R_{E \ell(\kappa)}(r) Y^{\left[\ell(\kappa) \frac{1}{2}\right] j(\kappa) m}(\theta, \varphi),
$$

where $Y^{\left[\ell(\kappa) \frac{1}{2}\right] j(\kappa) m} \equiv \chi_{m}^{\kappa}$ are the Pauli central field spinors. The quantum number $\kappa$ assumes the values $\pm k$. Applying the ladder operators (18) to Eq. (19) yields either zero or $\frac{\sqrt{2} \mathbf{S} \cdot \mathbf{A}_{0}}{k}$. Moreover, since $\{\mathcal{K}, \mathbf{S} \cdot \hat{\mathbf{r}}\}=0$ (see theorem) and $(\mathbf{S} \cdot \hat{\mathbf{r}})^{2}=\frac{1}{4}$, we obtain the nice property that

$$
(\mathbf{S} \cdot \hat{\mathbf{r}}) \chi_{m}^{ \pm k}=-\frac{1}{2} \chi_{m}^{\mp k}
$$

Using Eq. (17) and appropriate phase conventions, we find

$$
\left(\frac{\sqrt{2} \mathbf{S} \cdot \mathbf{A}_{0}}{k}\right) \phi_{E \pm k m}=-\left(E+\frac{1}{2 k^{2}}\right)^{\frac{1}{2}} \phi_{E \mp k m} .
$$

Hence states within the subspace $\mathcal{H}_{k}$, with fixed $E$ and $m$, transform irreducibly under the superalgebra $S(2)$. The multiplets have dimensionality two, unless $E=-\frac{1}{2 k^{2}}$, in which case they are one-dimensional.

Eq. (21) can now be reduced to a set of radial equations. Using the identity

$$
\mathbf{S} \cdot \mathbf{A}_{0}=\left[1-\mathcal{K}\left(i \hat{\mathbf{r}} \cdot \mathbf{p}+\frac{\mathcal{K}+1}{r}\right)\right](\mathbf{S} \cdot \hat{\mathbf{r}})
$$


and substituting Eqs. (20, 22) in Eq. (21) we find $(\kappa= \pm k)$

$$
\frac{1}{\sqrt{2} k}\left[1-\kappa\left(\frac{d}{d r}+\frac{\kappa+1}{r}\right)\right] R_{E \ell(\kappa)}(r)=\left(E+\frac{1}{2 k^{2}}\right)^{\frac{1}{2}} R_{E \ell(-\kappa)}(r) .
$$

Introducing furthermore $r R_{E \ell}(r)=\chi_{E \ell}(r)$ and identifying $k=\ell+1$, these two equations can be written as

$$
\left(\begin{array}{cc}
0 & A^{-}(\ell) \\
A^{+}(\ell) & 0
\end{array}\right)\left(\begin{array}{c}
\chi_{E \ell}(r) \\
\chi_{E \ell+1}(r)
\end{array}\right)=\sqrt{E+\frac{1}{2(\ell+1)^{2}}}\left(\begin{array}{c}
\chi_{E \ell}(r) \\
\chi_{E \ell+1}(r)
\end{array}\right),
$$

where

$$
A^{ \pm}(\ell)=\frac{1}{\sqrt{2}}\left[ \pm \frac{d}{d r}-\frac{\ell+1}{r}+\frac{1}{\ell+1}\right]
$$

The radial ladder operators $A^{ \pm}(\ell)$ are identical to those found in the radial SUSY studies of the Coulomb problem [2, 9]. We stress the fact that in those cases the angular momentum is not really affected, because the supercharges are purely radial. In our three-dimensional study the $\ell$-value $i s$ changed by the charges, as is demonstrated by Eq. (20).

We are now in a position to reinterpret the spectrum of a nonrelativistic spin- $\frac{1}{2}$ particle in a Coulomb field (see Fig. 1).

- The degeneracies of levels with fixed $j$ and $m$ but with $\ell=j \pm \frac{1}{2}$ are a consequence of the $S(2)$ supersymmetry algebra, constructed above. The degeneracy level is two, except for the states with $\ell=j-\frac{1}{2}$ and $E=-\frac{1}{2(\ell+1)^{2}}$, which are non-degenerate.

- The degeneracies of fixed $\ell$ and $m$ but $j=\ell \pm \frac{1}{2}$ are due to kinematic independence of the electron spin in the nonrelativistic regime. Ladder operators between these states can be constructed out of the vector operator $\mathbf{S} \times \mathbf{L}$ (see e.g. [10]). 
- The $(2 j+1)$-fold degeneracy of states with fixed $j$ and $\ell$ is due to rotational invariance of the interaction.

\section{Concluding remarks}

In this paper we have used the operators $\mathbf{A}_{0}, \mathbf{L}$ and $\mathbf{S}$ to construct the charges $Q_{1}$ and $Q_{2}$ of an $S(2)$ supersymmetry algebra of the nonrelativistic Coulomb problem. This supersymmetry occurs for all levels with fixed $j$ and $m$, as opposed to the $S O(4)$, which only concerns the bound states. The results derived in this paper, show that supersymmetry in the Coulomb problem can be extended beyond the one-dimensional formulation. It is not clear in general in how far this also applies to other physical systems, where supersymmetry is expected to hold. It is therefore of interest to study also these other cases. In particular, it may offer a possibility to explore the case

of a spin- $-\frac{1}{2}$ particle in a dyon field. The symmetry and spectrum-generating algebra of such a system are identical to those of the hydrogen atom [11]. It therefore seems reasonable to expect the $S(2)$ supersymmetry to hold as well.

As another object of interest, we mention the Dirac particle in a Coulomb field. The relativistic spin-orbit coupling breaks the degeneracies of equal $\ell$. However, as can be seen in Fig. 2, the relativistic hydrogen spectrum still exhibits the features of the $S(2)$ supersymmetry of equal $j$. Although this was also noticed by Sukumar [12], the corresponding supercharges have not yet been constructed. Our study strongly suggests that the symmetry group of the relativistic Coulomb problem is $S O(3) \times S(2)$, being the remainder 
of the $S O(4) \times S U(2)$ symmetry of the nonrelativistic problem. Clearly, it should be interesting to also find the explicit form of the supercharges in this case. If the Lamb shift, due to vacuum fluctuations, is taken into account, the $S(2)$ symmetry is broken as well, leaving only the rotational $S O(3)$. 


\section{References}

[1] A. Lahiri, P.K. Roy and B. Baghi, Int. J. Mod. Phys. A5, 1383 (1990).

[2] V.A. Kostelecký and M.M. Nieto, Phys. Rev. Lett. 53, 2285 (1984); Phys. Rev. A32, 1293 (1985).

[3] V.A. Kostelecký, M.M. Nieto and D.R. Truax, Phys. Rev. D32, 2627 (1985).

[4] C.V. Sukumar, J. Phys. A18, 2917 (1985); J. Phys. A18, 2937 (1985);

J. Phys. A19, 2297 (1986).

[5] A.B. Balantekin, Ann. Phys. 164, 277 (1985).

[6] E. D'Hoker and L. Vinet, Phys. Rev. Lett. 55, 1043 (1985).

[7] W. Pauli, Z. Physik 36, 336 (1926).

[8] L.C. Biedenharn and L.D. Louck; Angular momentum in Quantum Physics, Encyclopedia of Mathematics and its applications, AddisonWesley Publishing Company (1981).

[9] R.D. Amado, Phys. Rev. A37, 2277 (1988).

[10] L.C. Biedenharn and P.J. Brussaard; Coulomb Exitation, Clarendon Oxford (1965).

[11] E. D'Hoker and L. Vinet, Nucl. Phys. B260, 79 (1985); Lett. Math. Phys. 12, 71 (1986).

[12] C.V. Sukumar, J. Phys. A18, L697 (1985). 


\section{Figure Captions.}

Fig. 1 The nonrelativistic hydrogen spectrum. All levels of equal $j$ and $m$ (including those in the continuum) are connected by an $S(2)$ supersymmetry.

Fig. 2 The characteristic feature of $S(2)$ supersymmetry in the relativistic hydrogen spectrum (not to scale). 


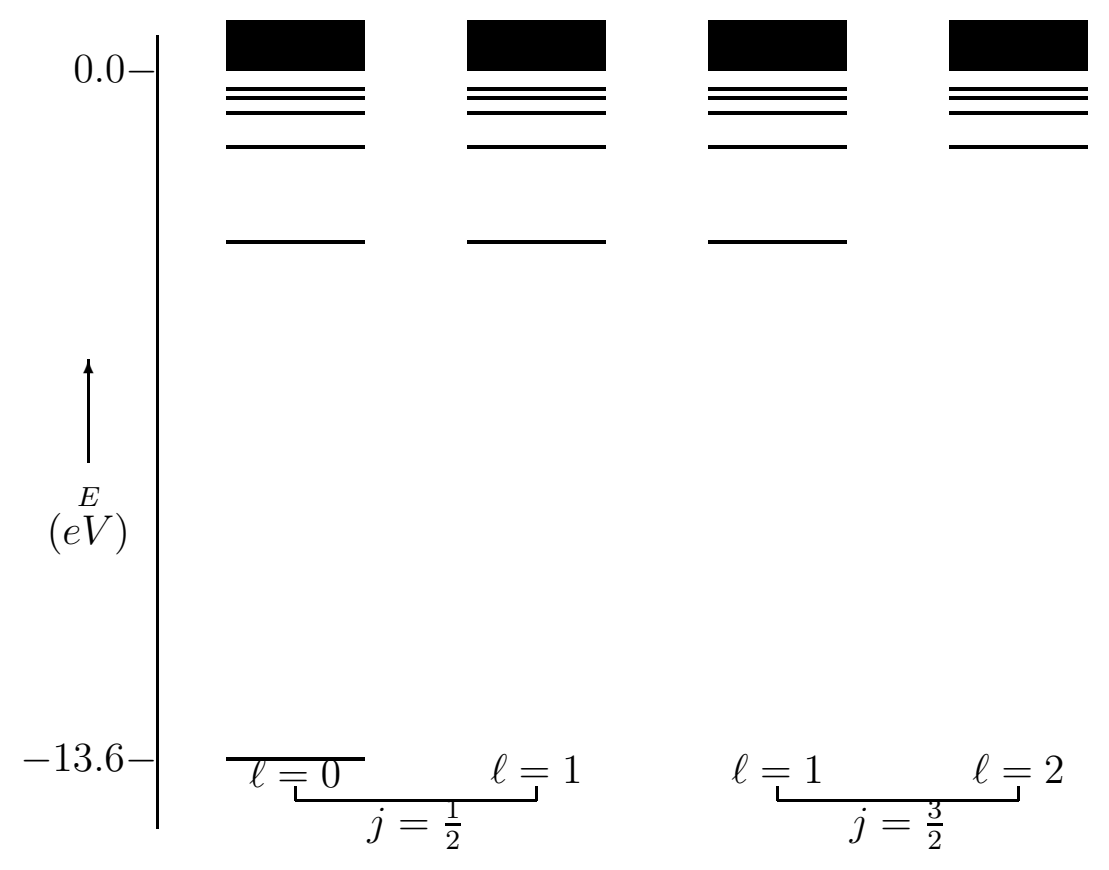

Figure 1. 


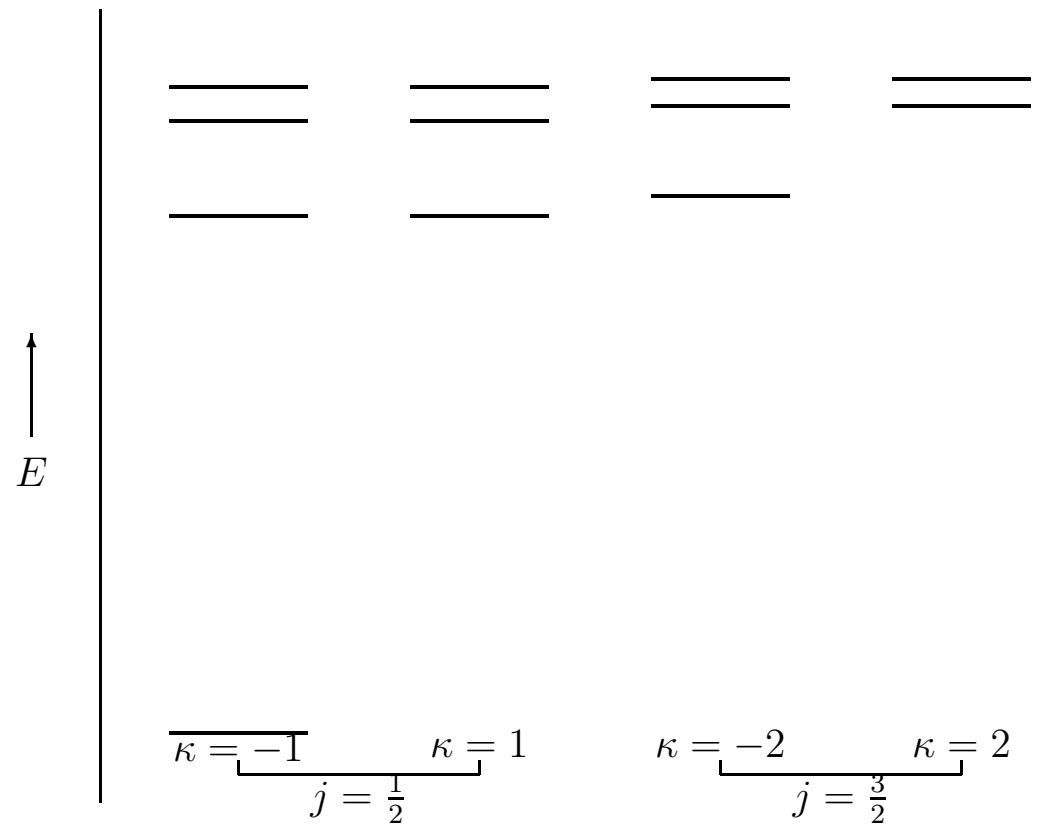

Figure 2. 